\title{
Surviving the Death Roll; Unveiling the Undiagnosed: Case of Hispanic Man with Covid-19 Pneumonia
}

\author{
Michael Khorsandi, MD*, Nirmala Manjappachar, MD, Elnaz Ebrahimihoor, MD and \\ Nargiz Muganlinskaya, MD, MMS, FACP
}

Department of Internal Medicine, Anne Arundel Medical Center, USA

*Corresponding author: Michael Khorsandi, MD, Internal Medicine Resident, Anne Arundel Medical Center, Luminis Health, 2001 Medical Parkway, Annapolis, MD 21401, USA, Tel: 832-759-8521, Fax: 443-949-7380

\begin{abstract}
Brugada syndrome $(\mathrm{BrS})$ is an inherited electrophysiological abnormality which typically manifests in patients with diverse ethnicities as ventricular arrhythmias or sudden cardiac death. The onset is frequently unmasked by various precipitating factors including but not limited to febrile diseases. Severe acute respiratory syndrome (SARS) coronavirus 2 (SARS-CoV-2) infection and associated COVID-19 illness continues to evolve. We are reporting a severe case of Covid-19 infection unveiling the undiagnosed Brugada syndrome, and the complicated course requiring mechanical ventilation and rounds of Cardiopulmonary resuscitation.
\end{abstract}

A 57-years-old Hispanic man with no known prior cardiac history presented to the emergency department with fever and altered mental status. Initial investigations revealed mild leukocytosis, and positive SARS-CoV-2 PCR. The patient was transferred to intensive care unit (ICU) due to hemodynamic instability and electrolyte derangements. On day 2 of ICU stay, patient became febrile, hypotensive, tachycardic, and his hypoxemia worsened with increased supplemental oxygen requirement. His electrocardiogram (ECG) was consistent with atrial fibrillation and covedtyped Brugada pattern. At the early hours of the subsequent day, patient developed first episode of asystolic cardiac arrest requiring cardiopulmonary resuscitations (CPR) and intubation leading to return of spontaneous circulation (ROSC) after 10 mins. Only 3 days after the initial cardiac arrest episode, patient suffered from another episode of asystolic cardiac arrest requiring CPR leading to return of normal sinus rhythm and ROSC. Patient had a successful recovery from COVID-19 pneumonia with no additional cardiac events during his hospitalization, and was subsequently extubated and discharged from hospital with close follow up monitoring.
Reported cases of Brugada syndrome in Hispanic population are scarce. Covid-19 positive patients have a guarded prognosis when they require intubation with mechanical ventilation with no chances of survival when they suffer cardiac arrest requiring cardiopulmonary resuscitation. This stimulated us to report a case of Brugada syndrome presented in Hispanic man who recovered a deadly course of Covid-19 infection despite requiring intubation and complicated course by two rounds of cardiopulmonary resuscitation (CPR).

\section{Keywords}

Hispanic, COVID-19, Brugada syndrome, Ventricular arrhythmias, Cardiac arrest, Sudden cardiac death

\section{Introduction}

Brugada syndrome (BrS) is an inherited electrophysiological abnormality syndrome which typically manifest in adulthood with mean age of $40 \pm$ 22-years-old at the onset of diagnosis [1,2] and appears as sudden cardiac death (SCD) up to $20 \%$ with no gross structural abnormalities of heart [3]. It has been attributed to SCN5A and SCN10A genes mutations in the cardiac sodium channel, which can affect repolarizationdepolarization abnormalities [4]. Brugada syndrome presents with ventricular arrhythmias and characteristic electrocardiographic (ECG) findings.

Despite the identified genetic abnormalities, the pathophysiological mechanism that causes lifethreatening ventricular arrhythmias and SCD in BrS remains controversial. It is understood that the trigger for arrhythmia is due to juxtaposition of myocytes with

Citation: Khorsandi M, Manjappachar N, Ebrahimihoor E, Muganlinskaya N (2021) Surviving the Death Roll; Unveiling the Undiagnosed: Case of Hispanic Man with Covid-19 Pneumonia. Int Arch Cardiovasc Dis 5:044. doi.org/10.23937/2643-3966/1710044

Accepted: October 13, 2021: Published: October 15, 2021

Copyright: (C) 2021 Khorsandi M, et al. This is an open-access article distributed under the terms of the Creative Commons Attribution License, which permits unrestricted use, distribution, and reproduction in any medium, provided the original author and source are credited. 
varying refractory periods and can be precipitated by fever, alcohol, cocaine dependence, psychotropic drugs, and tricyclic antidepressants. In this case report, we describe a patient who demonstrated type 1 Brugada ECG pattern (coved type ST-segment elevation $\geq 2 \mathrm{~mm}$ followed by a negative T-wave in $\geq 1$ of the right precordial leads $\mathrm{V}_{1}$ to $\mathrm{V}_{2}$ ) in the setting of hospitalization for COVID-19 pneumonia. Our patient survived multiple cardiac arrests with effective and timely resuscitations in the intensive care unit and was successfully discharged home.

\section{History of Presentation}

A 57-year-old Hispanic man was brought in to the Emergency Department (ED) by ambulance because of fever and progressive altered mental status, during the coronavirus disease 2019 pandemic (Covid-19), the disease caused by severe acute respiratory syndrome coronavirus 2 (SARS-CoV-2). Prior to arrival to our facility, patient was evaluated at a local urgent care clinic for auditory hallucinations where he was found to have multiple abnormal laboratory results. Upon arrival he had temperature of $97.9^{\circ} \mathrm{F}$, heart rate of 115 beats per minute, blood pressure of $101 / 47 \mathrm{mmHg}$ and respiratory rate of 22 per minute. Oxygen saturation was $94 \%$ while breathing ambient air. Patient was confused but he was able to report decrease in appetite and feeling of fatigued. Physical exam was significant for ill appearance, sclera icterus and jaundiced skin. He was tachypenic and had increased work of breathing but the rest of respiratory and cardiac physical exam was unremarkable. Patient was awake and oriented to self only. Neurological exam was significant for asterixis and delayed response when following simple commands.

\section{Past Medical History}

Patient's past medical history was notable for alcohol use disorder, with consumption of 18-20 beers per day, and intermittent intranasal cocaine consumption. Family member reported that patient had been abstinent for 2 weeks prior to the visit. There was no known history of smoking or drug allergies or any sudden cardiac death in the family.

\section{Differential Diagnosis}

Patient presented with fever in the midst of Covid-19 pandemic. We created a list of differential diagnosis such as infectious encephalitis (Including Covid-19), Sepsis, Metabolic encephalopathies (Hepatic, Renal, Electrolyte derangement), dehydration, Substance intoxication, alcohol withdrawal, and acute Intracranial pathology (traumatic or vascular accident).

\section{Investigation}

The initial laboratory values Table 1 demonstrated leukocytosis, hyponatremia, elevated BUN and $\mathrm{Cr}$,

Table 1: The initial laboratory values (on arrival to ED) in middle column; reference range (Right column).

\begin{tabular}{|c|c|c|}
\hline Laboratory data & On arrival to ED & Reference Range, Adults \\
\hline WBC & 28.8 & $4.80-10.8010 * 3 / \mu \mathrm{L}$ \\
\hline RBC & 4.88 & $4.50-5.9010^{*} 6 / \mu \mathrm{L}$ \\
\hline Hemoglobin & 14.9 & $13.5-17.1 \mathrm{~g} / \mathrm{dL}$ \\
\hline Hematocrit & 42.4 & $41.0-51.0 \%$ \\
\hline MCV & 86.9 & $81.0-99.0 \mathrm{fL}$ \\
\hline Neutrophils Absolute & 25.34 & $1.80-7.7010^{*} 3 / \mu \mathrm{L}$ \\
\hline Lymphocyte Absolute & 0.86 & $1.00-4.8010 * 3 / \mu \mathrm{L}$ \\
\hline Platelets & 129 & $150-35010 * 3 / \mu \mathrm{L}$ \\
\hline BUN & 52 & $5-25 \mathrm{mg} / \mathrm{dL}$ \\
\hline Sodium & 119 & $135-146 \mathrm{mmol} / \mathrm{L}$ \\
\hline Potassium & 3.8 & $3.2-5.0 \mathrm{mmol} / \mathrm{L}$ \\
\hline Chloride & 83 & $95-112 \mathrm{mmol} / \mathrm{L}$ \\
\hline $\mathrm{CO}^{2}$ & 25 & $18-32 \mathrm{mmol} / \mathrm{L}$ \\
\hline Glucose, Bld & 208 & $65-140 \mathrm{mg} / \mathrm{dL}$ \\
\hline Creatinine & 2.3 & $0.5-1.5 \mathrm{mg} / \mathrm{dL}$ \\
\hline Magnesium & 2.9 & $1.5-2.5 \mathrm{mg} / \mathrm{dL}$ \\
\hline Calcium & 7.4 & $8.1-10.2 \mathrm{mg} / \mathrm{dL}$ \\
\hline Anion Gap & 11 & $5-20 \mathrm{mmol} / \mathrm{L}$ \\
\hline AST & 107 & $10-40 \mathrm{U} / \mathrm{L}$ \\
\hline ALT & 73 & $4-44 \mathrm{IU} / \mathrm{L}$ \\
\hline Alkaline Phosphatase & 150 & $46-116 \mathrm{IU} / \mathrm{L}$ \\
\hline Bilirubin, Total & 6.6 & $0.3-1.3 \mathrm{mg} / \mathrm{dL}$ \\
\hline
\end{tabular}




\begin{tabular}{|c|c|c|}
\hline Bilirubin, Direct & 5.8 & $0.0-0.5 \mathrm{mg} / \mathrm{dL}$ \\
\hline Total Protein & 7.3 & $6.4-8.2 \mathrm{~g} / \mathrm{dL}$ \\
\hline Albumin & 2.2 & $3.2-5.0 \mathrm{~g} / \mathrm{dL}$ \\
\hline Ammonia & $<17$ & $17-80 \mu \mathrm{g} / \mathrm{dL}$ \\
\hline Acetaminophen Level & $>2$ & $\begin{array}{l}\text { Therapeutic range: } 10-30 \mu \mathrm{g} / \mathrm{mL} \\
\text { Toxicity level: }>200 \mu \mathrm{g} / \mathrm{mL} \mu \mathrm{g} / \mathrm{mL}\end{array}$ \\
\hline Ethanol, Serum & $<3$ & $0-10 \mathrm{mg} / \mathrm{dL}$ \\
\hline Lactate & 2.3 & $0.5-2.0 \mathrm{mmol} / \mathrm{L}$ \\
\hline Salicylate Lvl & $>2$ & $<20 \mathrm{mg} / \mathrm{dL}$ \\
\hline Troponin I & 0.496 & $0.000-0.045 \mathrm{ng} / \mathrm{mL}$ \\
\hline Barbiturate Screen, Ur & Negative & \\
\hline Benzodiazepine Screen, Urine & Negative & \\
\hline PCP Screen, Ur & Negative & \\
\hline Cannabinoid Scrn, Ur & Negative & \\
\hline Cocaine (Metab.) Screen, Urine & Negative & \\
\hline Opiate Screen, Urine & Negative & \\
\hline Methadone, Screen Urine & Negative & \\
\hline Amphetamine Screen, Ur & Negative & \\
\hline Prothrombin Time & 15.6 & $11.4-13.8 \mathrm{sec}$ \\
\hline INR & 1.3 & $1.0-1.0$ \\
\hline
\end{tabular}

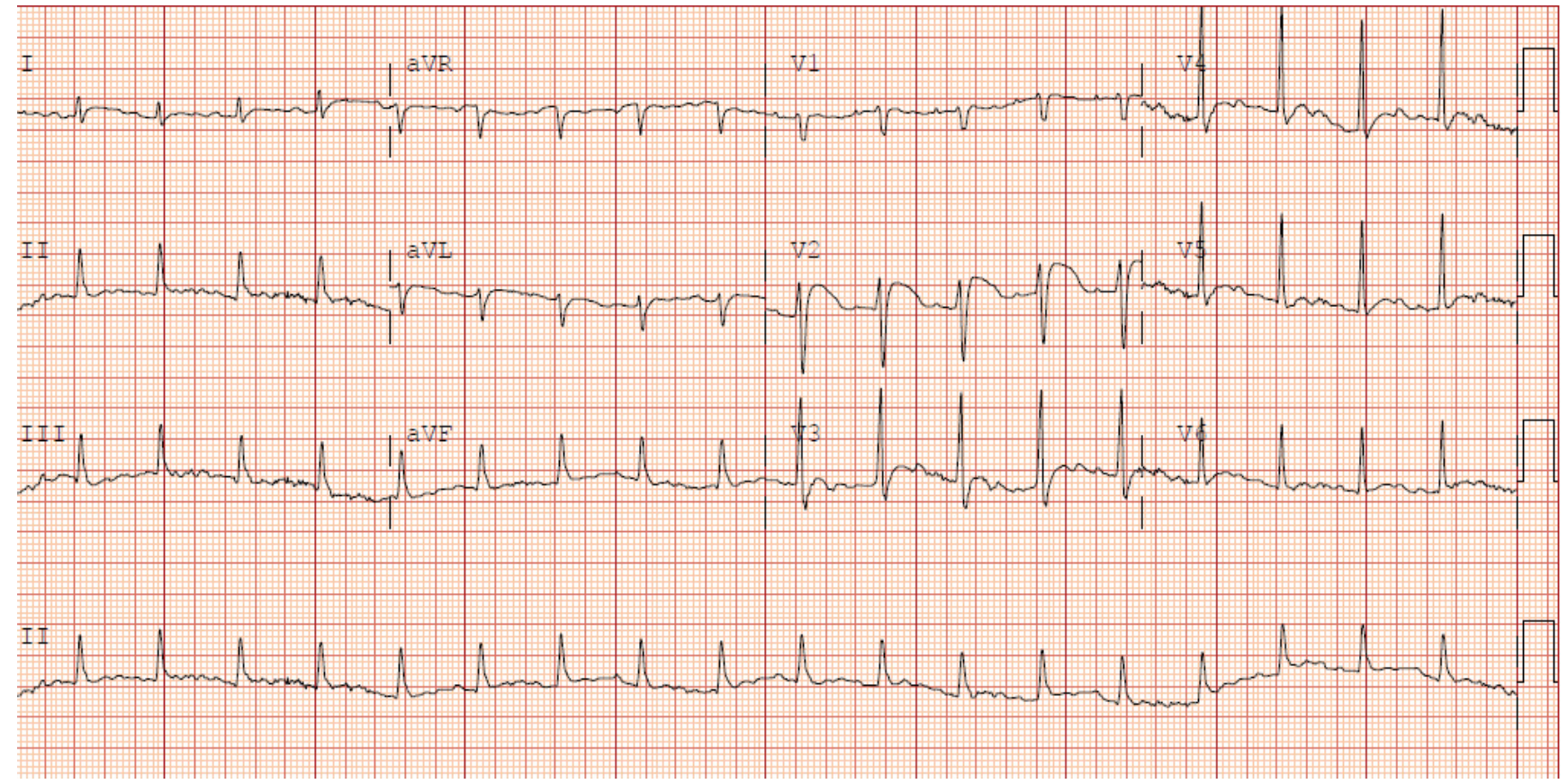

Figure 1A: Initial ECG showed normal sinus rhythm with heart rate of 112 beats per minute with nonspecific ST segment changes in lead V2.

deranged liver function test, Low ammonia level and slightly elevated Troponin. ECG showed normal sinus rhythm with heart rate of 112 beats per minute with nonspecific ST segment changes in lead V2 (Figure 1A). Urine toxicology screen was negative for alcohol, cocaine, amphetamine, barbiturates, opiates, PCP, and benzodiazepine. There were undetectable level of alcohol and salicylates on serum toxicology screen. Testing of the nasopharyngeal swab was positive for SARS-CoV-2 RNA. Computed tomographic (CT) of the head revealed no evidence of intracranial abnormality. $\mathrm{CT}$ of the chest-abdomen-pelvis (Figure 1B) revealed right suprahilar peribronchial ground-glass opacity, mild cardiomegaly, trace peripheral vascular congestion and mild hepatomegaly. There were no discernible peripheral ground-glass opacities to raise the concern for COVID19 pneumonia. Urinalysis was concerning for an infection. Blood cultures were sent for possible bacteremia. 


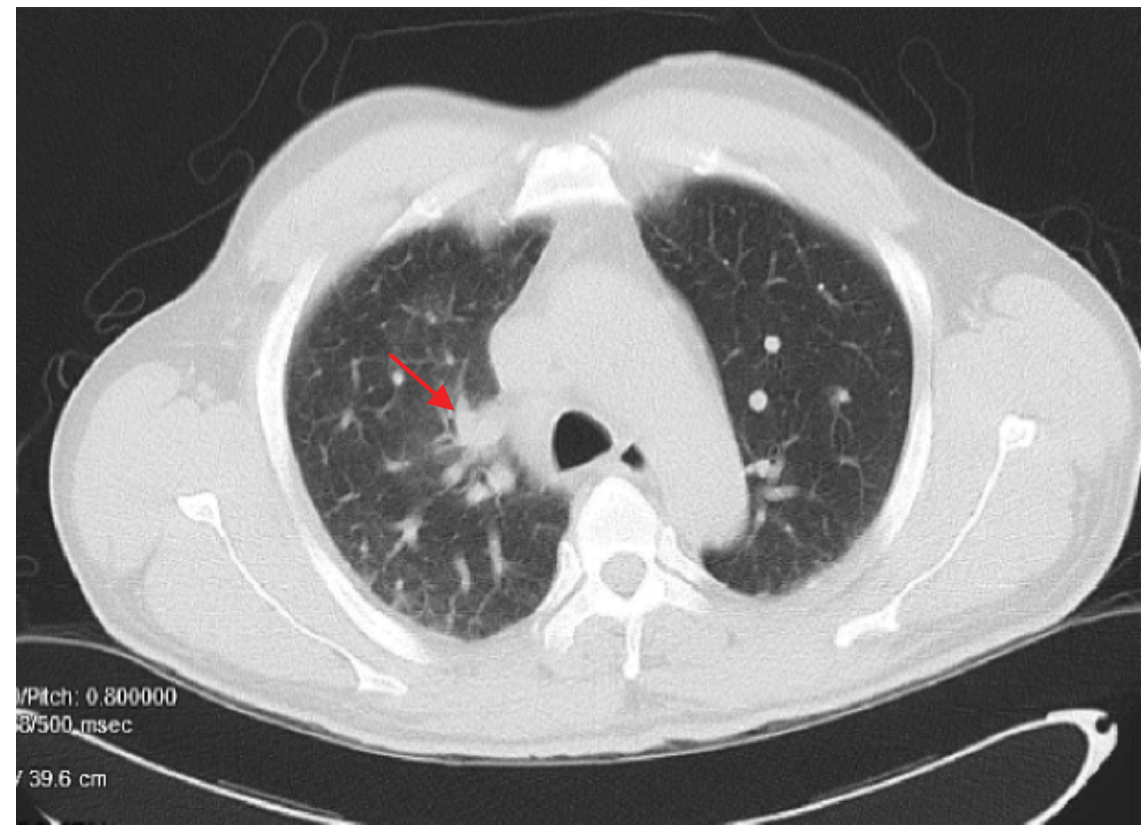

Figure 1B: CT of the chest-abdomen-pelvis revealed right suprahilar peribronchial ground-glass opacity (red arrow), mild cardiomegaly, trace peripheral vascular congestion and mild hepatomegaly.

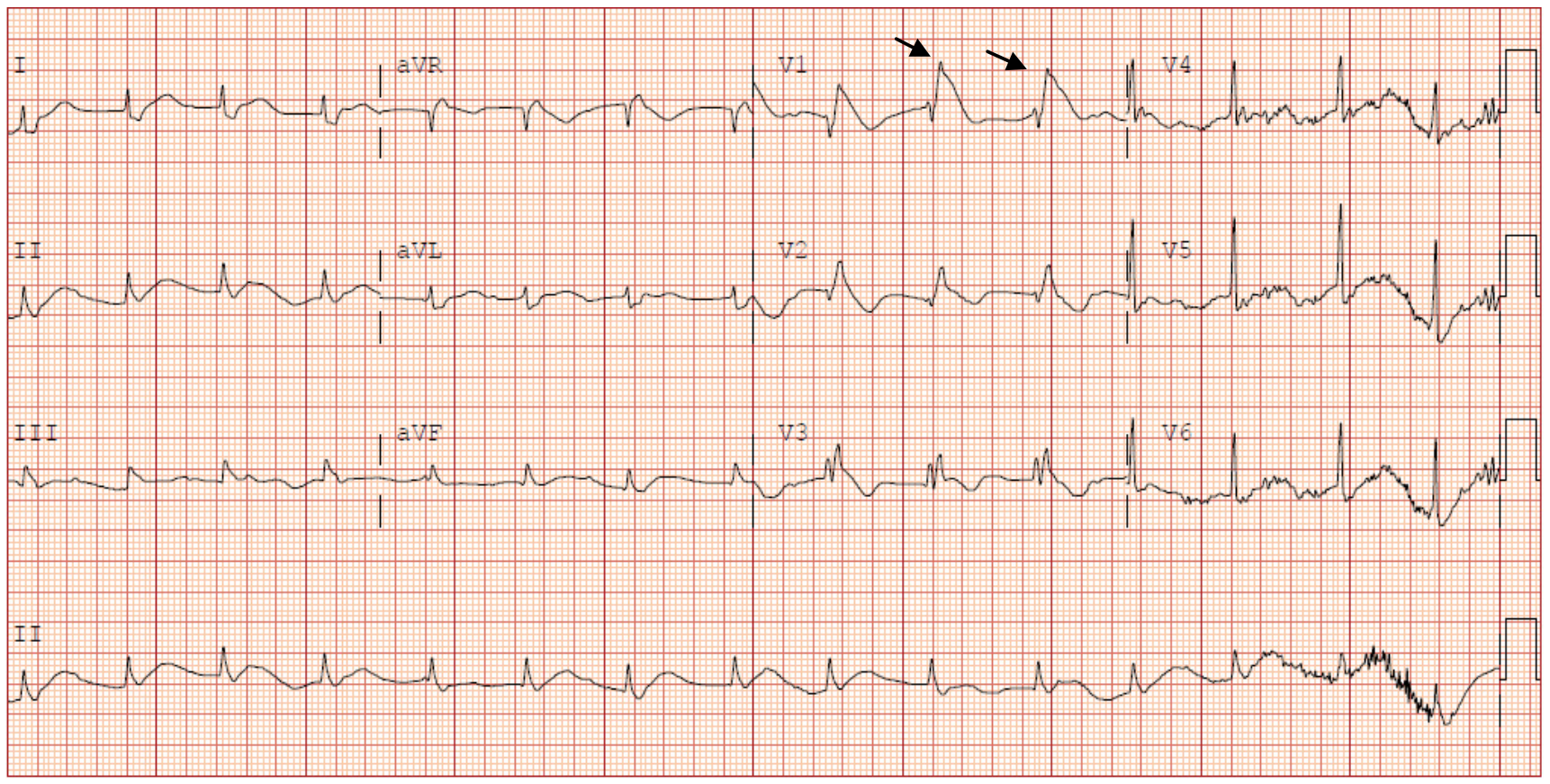

Figure 1C: Repeat ECG revealed new atrial fibrillation with the ventricular rate of 108 beats per minute with $>2 \mathrm{~mm} \mathrm{~J}$ point elevation in leads V1-V3 with gradually descending ST segment followed by a negative T wave consistent with Coved-typed Brugada pattern.

\section{Management}

While urine culture in process, patient was started on empiric intravenous ceftriaxone for presumed urinary tract infection. Given the history of alcohol use disorder, he was started on chlordiazepoxide, thiamine, and folic acid. Patient was placed under Contact and droplet Isolation for positive SARS-CoV-2 RNA test.

Shortly after arrival, patient was deemed to be hemodynamically unstable as he remained hypotensive (MAP in 60's) and non responsive to intravascular volume expansion. He was admitted to the intensive care unit (ICU) for the management of hyponatremia, hypotension and delirium. He was continued on intravascular volume expansion with crystalloids.

On day 2 of hospital stay patient developed fever of 101 of and hypotension persisted (mean arterial pressure $<65$ ). He remained tachypneic with the respiratory rate of 44 breaths per minute. He was supplemented with 2 litres of oxygen, delivered via nasal canula (with the goal of saturation $>94 \%$ ). IV Vancomycin and Piperacillin-Tazobactam were added 
empirically for presumed sepsis. Catheters were placed in the right femoral vein and the right radial artery, for close hemodynamic status monitoring.

Additional laboratory investigations were remarkable for elevated levels of Brain Natriuretic Peptide (BNP) more than $5000 \mathrm{pg} / \mathrm{mL}$, [reference range $0-100 \mathrm{pg} / \mathrm{mL}$ ] and elevated D-Dimer $3.44 \mu \mathrm{g} / \mathrm{mL}$ [reference range < $0.50]$. Repeat ECG revealed new atrial fibrillation with the ventricular rate of 108 beats per minute with $>2$ $\mathrm{mm} J$ point elevation in leads V1-V3 with gradually descending ST segment followed by a negative $T$ wave consistent with Coved-typed Brugada pattern (Figure 1C). Meanwhile Troponin peaked at 0.942 . Therapeutic anticoagulation with heparin was initiated. Patient was started on norepinephrine to support MAP above 65.

On day 3 of hospitalization, patient remained febrile with $\mathrm{T}$ max of $102{ }^{\circ} \mathrm{F}$ and tachycardia. At 2:30 am patient sustained an asystolic cardiac arrest. ACLS and BLS guidelines were applied and return of spontaneous circulation was attained within 10 minutes. Patient underwent endotracheal intubation followed by mechanical ventilation. He was placed on vasopressor support for hemodynamic support. Targeted temperature management was initiated for duration of 48 hours. Upon completion of targeted temperature management, neurological exam was notable for preserved Cough, Gag and corneal reflexes.

On day 6 of hospitalization, the patient suffered from another episode of asystole requiring chest compression with return of sinus rhythm. Subsequent transthoracic echocardiogram showed ventricular ejection fraction of $55-60 \%$ and grade I diastolic dysfunction of the left ventricle. There was mild enlargement of right ventricle and mildly decreased right ventricular systolic function. There were mild Mitral and tricuspid regurgitations.

Patient received standard of care for management of his COVID-19. Cardiac Telemetry monitoring continued throughout hospital stay. There was no additional cardiac event during hospitalization. On day 19 of hospitalization, patient was discharged from hospital in stable condition with no neurological sequelae with life vest and scheduled for follow up visit with his outpatient cardiologist for Implantable Cardioverter Defibrillator (ICD) placement.

\section{Discussion}

Ever since the first cases of COVID- 19 reported in 2019, there has been an overwhelming threat to public health at an epic scale. Its direct association with many diseases has remained an enigma.

Based on recent report from World Health Organization (WHO), since the initial detected cases of COVID-19 on December 30, 2019 through October 11, 2020, over 37 million COVID-19 cases and 1 million deaths have been reported globally [5]. Despite the increasing number of reported cases and substantially worse outcomes due to COVID-19 in certain racial and ethnic groups, much about the intricacies of this disease remains unknown.

In the setting of SARS-CoV-2, some of the cardiovascular complications that have been previously described are myocardial injury, myocarditis, acute coronary syndromes, and arrhythmias [6]. Wang D, et al. [7] reported that among 138 hospitalized patients with COVID-19, $16.7 \%$ had arrhythmias and $7.2 \%$ had acute myocardial injury. However, at present, the risk of worsening cardiovascular disease in patients with COVID-19 with underlying cardiovascular disease (CVD) remains unknown. In Covid-19 patients, the main determinants of a worse prognosis, including need for mechanical ventilation and death [8]. Reported In hospital mortality after inpatient cardiac arrest in Covid-19 patients regardless of baseline comorbidities is $100 \%$ [9].

Brugada syndrome is an extremely rare genetic disorder first described in 1992 with incidence of 0.05\% worldwide [10]. It frequently manifests with an increased prevalence in the Asian population, with Asians affected about 9 times more than Caucasians and 36 times more often than Hispanics [11]. Taking into consideration that Brugada syndrome is a rare genetic disease with an infrequent incidence in the Hispanic population, our patient presenting with Brugada syndrome amidst battling COVID-19 deserved to be reported.

The Hispanic population is the largest ethnic minority group in the United States, comprising nearly 60 million people [12]. Despite the fact that Hispanics constitute $18 \%$ of the total US population, this group accounts for an alarming $28.4 \%$ of cumulative US COVID-19 cases within a single known ethnic group reported to the Centers for Disease Control and Prevention (CDC) as of 20 May 2020 [13].

\section{Follow-Up}

Patient was given life vest upon hospital discharge. Two days after his hospital discharge, patient was seen by his outpatient cardiologist and was scheduled for Implantable Cardioverter Defibrillator (ICD). He was later seen by his outpatient cardiologist for follow up visit after ICD placement. The patient has had no hospital admissions after his ICD placement and is following up with his outpatient cardiologist every 6 months for routine checkups.

\section{Conclusions}

Covid-19 patients have a guarded prognosis when they require mechanical ventilation and their survival is unprecedented when they suffer cardiac arrest. This case is amongst first cases of Brugada syndrome presented in Hispanic patients who recovered a deadly course of Covid-19 infection despite requiring intubation 
and discharged from the hospital after a complicated course after two episodes of cardiac arrest requiring cardiopulmonary resuscitation (CPR). In addition, this case emphasizes that clinicians should be vigilant on prompt diagnosis of Brugada pattern in COVID-19 patients. Early recognition of Brugada syndrome in the setting of Covid-19 as may have a significant clinical impact on patients and their families in preventing sudden cardiac death.

\section{Learning Objectives}

- To acknowledge the need for early detection of the Brugada pattern on electrocardiogram.

- To highlight the importance of prompt treatment of the primary disease or disturbances that caused unmasking of the Brugada pattern.

- To understand Brugada syndrome as a potential cause of ventricular arrhythmia and sudden cardiac death.

\section{Funding}

No grants, contracts, and other forms of financial support.

\section{Disclosure}

There are no relationships with industry.

\section{Acknowledgement}

We thank Dr. William Clarence Maxted, MD (Department of Cardiology at Anne Arundel Medical Center) for his guidance and critical review during the preparation of this manuscript.

\section{References}

1. Antzelevitch C (2006) Brugada syndrome. Pacing Clin Electrophysiol 29: 1130-1159.

2. Antzelevitch $C$, Brugada $P$, Borggrefe $M$, Brugada J, Brugada R, et al. (2005) Brugada Syndrome: Report of the second consensus conference. Circulation 111: 659-670.

3. Bezzina CR, Barc J, Mizusawa Y, Remme CA, Gourraud JB, et al. (2013) Common variants at SCN5A-SCN10A and HEY2 are associated with brugada syndrome, a rare disease with high risk of sudden cardiac death. Nat Genet 45: 1044-1049.

4. Blok M, Boukens BJ (2020) Mechanisms of arrhythmias in the brugada syndrome. Int J Mol Sci 21: 1-20.

5. World Health Organization (2020) Coronavirus disease (COVID-19) pandemic.

6. Driggin E, Madhavan MV, Bikdeli B, Chuich T, Laracy J, et al. (2020) Cardiovascular considerations for patients, health care workers, and health systems during the covid-19 pandemic. J Am Coll Cardiol 75: 2352-2371.

7. Wang D, Hu B, Hu C, Zhu F, Liu X, et al. (2020) Clinical characteristics of 138 hospitalized patients with 2019 novel coronavirus-infected pneumonia in wuhan, china. JAMA 323: 1061-1069.

8. Van Gerwen M, Alsen M, Little C, Barlow J, Genden E, et al. (2021) Risk factors and outcomes of COVID-19 in New York City; a retrospective cohort study. J Med Virol 93: 907-915.

9. Priyank S, Hallie S, Ayodeji O, Yash J, Abigail C, et al. (2021) Is Cardiopulmonary resuscitation futile in coronavirus disease 2019 patients experiencing in-hospital cardiac arrest?* , Critical Care Medicine 49: 201-208.

10. Brugada P, Brugada J (1992) Right bundle branch block, persistent ST segment elevation and sudden cardiac death: A distinct clinical and electrocardiographic syndrome. A multicenter report. J Am Coll Cardiol 20: 1391-1396.

11. Nguyen A, Flores M, Tano V (2020) Brugada Syndrome: Presentation and management of the atypical patient in the emergent setting. Clin Pract Cases Emerg Med 4: 251-254.

12. (2020) Data.

13. (2020) CDC COVID Data tracker. 